\title{
ERAS programs in elderly patients: is there a limit?
}

\author{
Monica Millan ${ }^{1}$ (D) $\cdot$ Beatriz Espina-Perez ${ }^{1} \cdot$ Aleidis Caro-Tarrago $^{1} \cdot$ Rosa Jorba-Martin ${ }^{1}$
}

Accepted: 5 July 2018 / Published online: 12 July 2018

(C) Springer-Verlag GmbH Germany, part of Springer Nature 2018

\section{Dear Editor:}

We read with great interest the article by P. Tejedor et al. [1] on the benefits of an ERAS program in elderly patients undergoing colorectal surgery. They elegantly show that ERAS principles can be applied in patients $>70$ years of age, and found a reduction in overall and severe complications and length of stay.

There is no mention, however, of the ASA status or selection of the patients included in the ERAS program. Were the more frail patients excluded? Would a modified ERAS protocol also be useful in frail, older patients?

Several studies have attempted to answer this question. A recent randomized, controlled trial used a geriatric review followed by a tailored intervention based on the results, in all elderly patients already included in an ERAS program, but did not find significant differences in postoperative complications between the groups [2]. Other authors, however, have found improvement in short-term results in frail patients after implementation of a multidisciplinary team approach [3].

Our group has been using a tailored, combined prehabilitation and ERAS program for patients $>70$ years of age since 2016. This program starts as soon as the diagnosis of colorectal cancer is made in the endoscopy suite. A specialized nurse then coordinates a frailty and/or geriatric evaluation and personalized prehabilitation according to the patient's characteristics. The ERAS program is tailored to the patient, and adherence is not considered an end-point. A liaison with elderly care physicians and specialized nurses and chronic care units is also used according to the patient's needs.

We compared a cohort of 75 patients included in this program between 2016 and 2017 with a matched cohort of 75 patients operated on before implementation of the program (2014-2015), and found that, although the mean ASA was III, polypharmacy was $52 \%$ in both groups ( $>5$ medications/ daily), and VES-13 was $>2$ in $61 \%$, there was a significant reduction in length of stay (mean 6 vs 9 days $(p<0,001)$, with no increase in readmissions. We also found a significant reduction in postoperative ileus $(13.3 \%$ with the program vs $29.3 \%$ before the program, $p=0,017)$, delirium ( 2.7 vs $20 \%$, $p=0.002)$, wound infection ( 8 vs $21.3 \%, p=0.02)$ and other complications (pneumonia, urinary tract infection, venous thrombosis: $1.3 \%$ with the program vs 22.75 before the program, $p<0,0001)$.

We believe that many frail, elderly patients, which are sometimes excluded from ERAS programs, can also benefit from a combination of a personalized prehabilitation program and many of the items of ERAS; this could add to the improvement in short-term results in this vulnerable group of patients, and we hope to see more of them included in future studies.

\section{References}

1. Tejedor P, Pastor C, Gonzalez-Ayora S, Ortega-Lopez M, Guadalajara H, Garcia-Olmo D (2018) Short-term outcomes and benefits of ERAS program in elderly patients undergoing colorectal surgery: a case-matched study compared to conventional care. Int $\mathbf{J}$ Color Dis. https://doi.org/10.1007/s00384-018-3057-z

2. Ommundsen N, Wyller TB, Nesbakken A, Bakka AO, Jordhøy MS, Skovlund E, Rostoft S (2018 Jan) Preoperative geriatric assessment and tailored interventions in frail older patients with colorectal cancer: a randomized controlled trial. Color Dis 20(1):16-25. https://doi. org/10.1111/codi.13785

3. Castellví Valls J, Borrell Brau N, Bernat MJ, Iglesias P, Reig L, Pascual L, Vendrell M, Santos P, Viso L, Farreres N, Galofre G, Deiros C, Barrios P (2018) Colorectal carcinoma in the frail surgical patient Implementation of a work area focused on the complex surgical patient improves postoperative outcome. Cir Esp 96(3):155161. https://doi.org/10.1016/j.ciresp.2017.09.015
Monica Millan

millanmonica@hotmail.com

1 Department of Surgery, Joan XXIII University Hospital, Tarragona, Spain 